\title{
Urgences
}

\section{Au centre du monde}

\section{Frédéric-Jacques Temple}

Numéro 17-18, octobre 1987

L'esprit des lieux

URI : https://id.erudit.org/iderudit/025416ar

DOI : https://doi.org/10.7202/025416ar

Aller au sommaire du numéro

Éditeur(s)

Urgences

ISSN

0226-9554 (imprimé)

1927-3924 (numérique)

Découvrir la revue

Citer ce document

Temple, F.-J. (1987). Au centre du monde. Urgences, (17-18), 12-18.

https://doi.org/10.7202/025416ar

Ce document est protégé par la loi sur le droit d'auteur. L’utilisation des services d'Érudit (y compris la reproduction) est assujettie à sa politique d'utilisation que vous pouvez consulter en ligne.

https://apropos.erudit.org/fr/usagers/politique-dutilisation/
Cet article est diffusé et préservé par Érudit.

Érudit est un consortium interuniversitaire sans but lucratif composé de l’Université de Montréal, l'Université Laval et l'Université du Québec à Montréal. Il a pour mission la promotion et la valorisation de la recherche. https://www.erudit.org/fr/ 
Premier atelier: 2 juin: $13 \mathrm{~h} 30$

Frédéric-Jacques Temple

Domingo Cisneros

Luc Bureau

René Derouin 


\section{Frédéric-Jacques Temple AU CENTRE DU MONDE}

Je viens d'un pays que borde la mer, et ce pays porte d'abord le nom de cette mer qui fut autrefois le berceau des dieux: la Méditerranée, la mer d'entre les terres. Je suis de cette vaste province dont les phares sont Gênes, Barcelone, Naples, Athènes, Alger ou Marseille, villes qui flambent sous le soleil. De là, Strasbourg ou Calais me sont exotiques, tout comme cette admirable capitale qui m'impose ses jardins à la française.

Dans mon enfance tendue vers la mer (car c'est elle que nous regardions, tournant le dos aux montagnes centrales) j'ai moins entendu parler de Paris que d'Aigues-Mortes, des Francs que des Sarrasins, de l'évèque de Reims que des Cathares. La saumure, l'ail, l'huile, la poutargue, le cade, le safran, ont façonné mon palais, éduqué mon odorat, bruni ma peau, aiguisé mes sens. Sur ces rivages, on parle encore, dans les campagnes et les très vieux quartiers des villes, une langue que Dante fut près de choisir pour écrire La Divine Comédie, qui a survécu tant bien que mal en dépit des interdits, des pressions, du centralisme; mon père la parlait; je puis encore la lire et la comprendre, même si je ne l'écris pas. Elle irrigue celle que j'utilise, l'ensemence, lui donne sa couleur. Le français que j'écris ou que je parle n'est pas tout à fait celui que j'entends en Bretagne, en Bourgogne, en Wallonnie ou au Québec. C'est précisément parce que je vis de mes singularités (et vous des vôtres), que je m'ouvre davantage au monde, assuré que Londres, Madrid ou New York n'exercent sur moi aucune tutelle pondéreuse. De ma ville, de ma garrigue de ronces et de chênes-verts, je peux converser librement avec le monde sans craindre de voir se dresser le spectre pourri du jacobinisme, sans la moindre infériorité, le moindre malaise à se juger vassal, ou l'humiliation de voir les provinces accourir quand les Paris sifflent. De ma ville, je dialogue d'égal à égal avec Rome, Vienne, Amsterdam ou Rimouski. Et s'il est évident que ma seule culture ne me suffit pas (car l'ouverture exclut toute limite) ce l'est aussi qu'aucune culture où la mienne ne serait pas admise ne me conviendrait. S'il est vrai que le poème n'a pas d'autre lieu que le poème, il n'en est pas moins fondamental que les racines de ce poème s'abreuvent à des infrastructures où fermente le génie du lieu, cet esprit des lieux qui fait que certains mots ne peuvent être que d'ici et non de là. Le langage n'est pas "cosa mentale». Les mots ont leur histoire, leur généalogie, leur odeur. Nés des choses, ils engrossent les choses. Ce n'est pas à vous québécois qu'il convient d'expliquer la phrase de la philosophe Simone Weil concernant un peuple: «lls avaient même un mot pour désigner la patrie, ils l'appelaient le langage». 
Mais précisément, ce point marqué sur le globe, où je vis, où j'écris, d'où je parle aux autres, est, si petit, si humble soit-il, le centre du monde. Ainsi placé, je suis reçu comme parlant pour tous de mes racines et de mes dieux. Mais parler au monde dans sa totalité, je ne peux le faire que pour autant que ma terre personnelle, mon terreau si vous voulez, me donne la force nécessaire à ce saisissement global de l'univers. Mon expérience singulière, mon aventure sur mon propre territoire, mon attention aux battements de son coeur, tout cela qui est mon domaine, je le donne à la planète, et je reçois de même d'elle.

Qu'est donc mon expérience d'homme, d'écrivain, sur un territoire donné? Longtemps, ce fut pour moi, dans l'enfance et l'adolescence, la parfaite inconscience, la totale irresponsabilité. Je ne savais pas encore que l'enfance était un lieu destiné à devenir mythique, de par sa nature même, un mythe qui, plus tard, me serait étranger lors du passage à l'état adulte qui est un état d'aliénation. Rien ni personne ne m'avait prévenu, quand j'étais au Paradis, que celui-ci était, par définition, destiné à être perdu. Beaucoup plus tard la prise de conscience que le Paradis était une "tour abolie", détermina chez moi le désir d'y pénétrer. Blaise Cendrars eut beau me dire: «ll ne faut jamais revenir au jardin de son enfance qui est un Paradis perdu", il était trop tard pour que je ne commisse pas la faute. Le temps avait fait son oeuvre. Et d'ailleurs, comment croire ces écrivainsconseilleurs qui n'ont jamais fait qu'enfreindre leurs propres résolutions? Dès lors que j'eus pénétré dans ce jardin interdit qui avait été le mien autrefois - cet autrefois qui est aussi un autre part - je me suis vite retrouvé en situation d'exil, car l'enfance est un pays lointain que nous portons en nous comme une hypothèque. De là à considérer le Sud comme une enfance perdue, parce qu'il est le lieu de cette enfance, il n'y avait qu'un pas que j'ai franchi en écrivant mon premier roman, Les eaux mortes'. Le Sud est donc devenu pour moi une certaine idée du Sud, non point tant un pays géographique qu'un pays profond qui est, comme l'a dit Gaston Bachelard, "moins une étendue qu'une matière".

Et nous voici du Côté de chez Swan où Marcel Proust, qui a reçu de Bergson les effluves de Matière et mémoire, entend "la rumeur des distances traversées", fort que selon Bergson "la vérité est que nous n'atteindrons jamais le passé sị nous ne nous y plaçons pas d'emblée». Ce passé est à la fois le «jamais plus» et le «toujours là", présents dans l'objet qui contient la mémoire. Nous savons que Proust trouve 
un végétal, une chose inanimée, perdues en effet pour nous jusqu'au jour, qui pour beaucoup ne vient jamais, où nous nous trouvons passer près de l'arbre, entrer en possession de l'objet qui est leur prison. Alors elles tressaillent, nous appellent, et sitôt que nous les avons reconnues, l'enchantement est brisé. Délivrées par nous, elles ont vaincu la mort et reviennent vivre avec nous. Il en est ainsi de notre passé. C'est peine perdue que nous cherchions à l'évoquer, tous les efforts de notre intelligence sont inutiles. Il est caché hors de son domaine et de sa portée, en quelque objet matériel (en la sensation que nous donnerait cet objet matériel) que nous ne soupçonnons pas. Cet objet, il dépend du hasard que nous le rencontrions avant de mourir, ou que nous ne le rencontrions pas.

Ainsi le Sud, ou l'enfance, peuvent-ils être enchaînés à un remous de la mer, au mouvement du vent, à une grappe de raisins, au cri d'une chouette au milieu de l'été; et nous voici dans le "jamais plus-toujours là», contemporain des cimetières qui sont la patrie essentielle.

Les ombres nous entourent, formant un royaume dont les cercueils ne sont que les apparences, et les superbes mausolées les dérisoires signes extérieurs d'une richesse abolie. Les ombres affirment leur présence, impalpables. Quiconque entre, aux portes d'une ville, dans cette ville aussi qu'est la nécropole, trouve un forum où les morts, au-delà des réalités qui travaillent sous les dalles, se meuvent par milliers, cohortes et légions invisibles entre les arbres et les stèles, régies par des lois qui nous sont étrangères, univers aux dimensions insondables qui sera le nôtre un jour, inexorablement. Là, demeure cachée aux yeux, mais présente comme par illumination secrète des cinq sens, l'âme collective d'une ville, d'un pays, dont le grand corps gît, dispersé mais entier, sédimentation millénaire. Et lorsque je pense à ma terre, je sens remuer doucement son grand cadavre, comme j'entends toujours battre furieusement les pompes spasmodiques du coeur sauvage de mon squale sur la grève écrasée de ciment.

Le squale dont il s'agit dans ce passage de Un cimetière indien², mon deuxième roman, est le héros d'une histoire bien réelle. C'est aussi l'exemple typique de la transmutation en fable, en mythe et en parabole d'un fait divers personnel qui revient prendre sa place, d'une autre manière, et avec une force nouvelle, clairement symbolique, plus de quarante ans après. Voici le récit:

Un jour que je me baignais après avoir relevé les filins, $j$ 'aperçus dans les vagues déferlantes l'aileron d'un requin chassant la mélette. Je courus vers la plage où toujours je tenais en réserve une foëne, et l'engin en main, je m'enfonçai dans l'eau jusqu'à la poitrine. La mer était d'une tiédeur ensorcelante, sous un ciel d'une tendre couleur de lavande fraîche. Le squale virevoltait - je voyais son aileron dorsal en lame de faux - se jouant des remous, tirant parti des plus hautes crêtes et des creux effervescents. Soudain, il fut sur moi. Sans attendre, je lançai mon arme qui se ficha dans le flanc de la bête, renversée pour l'attaque. Du ventre ivoirin et lisse fusa un jet de sang noir qui sema dans la mer des violettes. J'entendis claquer ses mâchoires barbelées et je vis, de part et d'autre de 
son groin, de petits yeux sauvages qui m'épiaient, luisant d'une couleur haineuse. De sa gueule à cinq rangées de dents aiguisées, s'échappaient des rubans de sang. Il battait furieusement l'eau de sa queue puissante. Agrippé au cordeau qui me reliait à la foëne, je maintenais ma prise, soucieux de ne pas la perdre, attentif à parer toute ruée. De son côté, le requin bondissait, disparaissait entre deux vagues, tentait de gagner le large. Il me fallait surtout ne pas céder d'un pas, garder le contact avec le sable, m'arc-bouter au mieux sur mes jambes. Le filin se tendait souvent à la limite de la rupture, parfois mollisait à l'extrême comme s'il était cassé. Il y avait de brefs instants d'indicible violence, et des accalmies dangereuses où j'ignorais si la bête avait de noirs desseins, si elle se préparait à charger ou avait réussi à se libérer du fer. Pied à pied, je gagnai enfin la plage: libre de mes mouvements, je pouvais mener le combat à ma guise. Au bout d'une demi-heure de lutte épuisante, j'échouai le squale qui avait faibli, mais dont l'énergie sauvage suffisait pour qu'il pût d'un coup de queue se projeter en l'air et battre le sable avec colère. Je ne pouvais détacher mon regard des petits yeux rageurs qui guettaient tous mes gestes, et j'admirais cette haine montant à travers sa nature profonde, cette malédiction muette proférée au nom de la vie, les forces obscures survoltant le sang qu'il avait encore à répandre sur le sable, et cette volonté primordiale capable de se prolonger au-delà de la mort. L'animal, enfin, cessa de bouger, et tout son être après un long spasme qu'on eut dit de jouissance, se figea... J'arrachai le coeur, gros comme un oeuf de canne, qui pompait avec une vigueur spasmodique. Je l'ensablai profondément avec les viscères et la chair inutilisable...Vers le soir, le vif désir me prit de revoir ce coeur sauvage. Il remuait encore. Je l'enfouis et le déterrai à plusieurs reprises. Il palpitait toujours... La nuit, sous la tente, je croyais entendre cogner régulièrement, avec le mien, le coeur turgescent et têtu qui ne voulait pas mourir.

Après avoir cité cet épisode, il convient de présenter le héros de mon récit. Je l'ai nommé Maleterre, allusion transparente au pays perdu et dégradé (mal à la terre, et la mauvaise terre), miroir dans lequel il ne se reconnaît plus, qui n'est plus conforme à ses réalités mythiques, un pays qui n'existe plus et qu'il s'agit de retrouver. Pour Maleterre, ce Sud-là ne serait-il pas un avatar du Graal? Mais sans doute aussi, le Sud est-il un ailleurs où il soit possible de se refaire une identité. Évoquer le Sud dans ce livre était donc, pour moi, me reconnaître comme aliéné, me situer en exil, puisque Maleterre s'expatrie d'un lieu élu, en l'occurence le Nouveau-Mexique. Là-bas, il imagine qu'il trouvera, parmi les Indiens Pueblos, à travers aussi le vieux rêve américain qu'il a muri dans son enfance, cet ailleurs, cet autre Sud, vécu par sa mémoire, et l'identité nouvelle conforme au passé «toujours là». Un soir, cheminant le long du Rio Grande pour rejoindre sa maison proche du désert, il entendra dans le lointain l'aboi prolongé d'un coyote, et comme le battement sourd d'un tambour dans un pueblo:

Maleterre écoute résonner ces coups, imaginant que le coeur de ce pays cogne sous la terre et se répercute entre les falaises du Rio Grande... Par delà les continents et les mers, entend-il aussi cet autre coeur palpitant 
sous le sable, un coeur qui n'en finit pas de mourir, frappant avec rage au milieu du cauchemar qui l'emprisonne, un coeur que j'ai enterré voilà bien longtemps - geste maintenant qui me hante où je vois une prémonition - le coeur sanglant, que je confonds avec celui de mon pays, du squale harponné un beau matin de septembre...

Un autre épisode de Un cimetière indien relate une chasse au puma dans les montagnes, et devient, lui aussi, prétexte à des remarques sur la dégradation, la disparition des images du vieux miroir. Maleterre rencontre, une semaine après la chasse, son ami John La Rivière, auteur du coup de fusil qui a tué le puma, et qui lui confie:

\begin{abstract}
J'ai bien compris ton amertume. Je sais ce que tu éprouvais devant le cadavre du puma... Je dois donc te faire un aveu. Ce puma, je l'ai tué avec passion, avec une sorte de frénésie panique. Le vieil instinct de la chasse, bien sûr, et de la possession, mais aussi le sentiment convaincu de contribuer à l'inéluctable; la certitude que tout était déjà réglé, qu'il fallait désormais que le destin se déroule jusqu'à son terme. Un jour nous ne serons plus là, ni les Indiens, ni personne qui ait pu connaître, comme nous, une terre encore semblable à celle qui existait voilà cinq mille ans. Tout va très vite maintenant, l'inévitable progresse comme un cancer. Nous sommes foutus, mon vieux, toi, moi, et les Indiens. Nous appartenons tous à un monde révolu. Les hommes nouveaux qui se sont installés un peu partout sont d'une autre race, qui n'est ni blanche, ni noire, ni rouge, ni jaune. C'est une race mentale. Ils ne vivent plus au rythme ancien du sang et de la sève, ils n'ont plus aucune intimité avec la nature. Ils parlent de nouveaux dieux qu'ils nomment ordinateurs, rentabilité, exploitation, consommation, statistique, prospective, marketing. Leur vocabulaire ne doit plus rien aux semences, aux sources, à la course du pollen dans le vent, à la migration des oiseaux, aux odeurs souterraines messagères des saisons, à tous ces signes désormais oubliés que la nature faisait à l'homme... Ils étaient assis dans un petit café mexicain, regardant le soleil se coucher derrière les arbres et les maisons d'adobe, buvant de la téquila pour tromper leur amertume, ou l'alimenter peut-être... Et je les rejoignais dans la tiédeur du crépuscule où le même soleil s'éteignait sur des terres dénaturées, les miennes, celles de Maleterre: on y avait depuis longtemps tué les pumas de l'enfance. Je me surpris à leur parler, à leur rappeler que, déjà, celui qui m'avait l'un des premiers, conduit par la main à travers les forêts du Nouveau-Monde, sur les traces des Natchez, avait reconnu que "dans un coeur troublé par le souvenir, il n'y a pas de place à l'espérance».
\end{abstract}

Ce double aspect de l'enfance dépossédée que forment l'exil et l'aliénation, était déjà fortement présent dans le roman dont j'ai cité le titre en commençant: Les eaux mortes. L'homme qui, après la guerre, revenait dans sa ville natale n'y retrouvait pas son propre visage. Face aux cariatides qui ornaient les maisons de la grande place, son interrogation était vaine:

Les femmes muettes sous les balcons n'ont pas voulu sourire. Il était trop tard maintenant pour espérer qu'elles s'y décident. Elles demeurent aujourd'hui momies de pierre déterminées dans leur figement, pour me rappeler ce temps couché sur ma mémoire, telle une dame immense 
pesant sur la tombe où gisent les reliques d'un enfant mort. Et je fus cet enfant dont je suis la tombe. Je le porte en moi dont il ne sortira jamais, ce moi-même étranger devenu mon fruit mort, mais qui remue sans espoir de naissance. Je suis devenu ma propre mère, à la matrice sans issue.

Que restait-il donc à faire? Maleterre s'interroge. Un Indien lui donne la réponse: "Tu es de la terre qui t’a fait naître, tes dieux sont là-bas où le soleil se lève, même si ce pays n'est plus qu'une réserve, ton cimetière indien". Maleterre se rend à l'évidence:

\begin{abstract}
Je vais donc revenir au pays. Peut-être aurai-je la chance d'y reconnaître des hommes de l'antique race égarés dans la foule des mutants installés sur les vestiges d'un monde perdu. J'imagine, à travers un brouillard, ce qui m'attend là-bas, dans la petite maison où les objets poussiéreux, les livres moisis, les meubles ternis vont m'agresser. Je pourrai, au moins, aller au cimetière pour retrouver mes racines, et découvrir ça et là, parmi les rues oubliées d'une ville fantôme quelques reliques solitaires. Je remettrai ma veste de futaine noire. Je serai démodé. Personne ne me reconnaitra, je l'espère. Assis sur le pas de la porte, à l'ancienne manière, j'attendrai mon passé, en fumant la pipe, sous le micocoulier...
\end{abstract}

Quand l'esprit des lieux est mort, quand les lieux eux-mêmes s'altèrent et meurent, que reste-t-il du bonheur? Celui-ci ne serait-il donc désormais que la suave, l'exemplaire médiocrité?

L'an dernier, j'ai vu s'ébattre, le long du Saint-Laurent, au cap Tourmente, des milliers d'oies blanches. Ces beaux oiseaux cacardaient à l'envi. Je me disais que, par eux, j'accédais à l'esprit de votre pays, que je lui reconnaissais un visage, ce même visage qui m'apparaît dans la blondeur des érables en hiver, dans le noir profond des épinettes sur la neige ou la splendeur de cuivre et d'or du feuillage d'automne. Là se trouvent vos dieux qui parlent à travers vos poètes et vos peintres.

Je forme le voeu que ces dieux qui sont les vôtres, je puisse longtemps encore les rencontrer au détour d'une piste, au bord de vos mille lacs, et qu'ils me disent qui vous êtes, comme les miens me disent qui je suis.

Écrire sur sa terre, même si c'est comme Job sur son fumier, c'est se retrouver dans les méandres d'une rivière ou dans les traces du vent sur le sable, dans le vol des bernaches ou dans la plainte lointaine d'un loup. Il est bien des façons d'être sur la plantète, d'y écouter l'esprit des lieux. Encore faut-il y être pleinement soi-même et que cela vous soit permis.

Dans mes garrigues et mes étangs du Sud, dans vos forêts sans frontière et sur vos grands fleuves, nous menons le même combat, 
nous poursuivons la même quête. Et à travers ce qu'il faut bien appeler le désespoir de ne pas se reconnaître dans le miroir terni du Paradis perdu de l'enfance, nous gardons l'espérance que l'avenir nous préservera de l'irréversible pourrissement de notre âme.

\section{NOTES}

1. Frédéric-Jacques Temple: Les eaux mortes, Albin-Michel, Paris 1975.

2. Frédéric-Jacques Temple: In cimetière indien, Albin-Michel, Paris, 1981. 